\title{
Single-center study: dynamic contrast-enhanced ultrasound in the diagnostic assessment of carotid body tumors
}

\author{
Vincent Schwarze, Constantin Marschner, Giovanna Negrao De Figueiredo, Michael Ingrisch, Johannes \\ Rübenthaler, Dirk-André Clevert \\ Department of Radiology, University Hospital, LMU Munich-Grosshadern Campus, Munich, Germany
}

Correspondence to: Vincent Schwarze. Department of Radiology, University Hospital, LMU Munich-Grosshadern Campus,Munich, Marchioninistrasse 15, 81377 Munich, Germany. Email: vincentschwarze@gmx.de.

Background: Carotid body tumors (CBTs) depict the most common paraganglioma of the head and neck that can metastasize in up to $15 \%$ of cases. They develop either sporadically or hereditarily and may produce catecholamines ("functioning CBTs") resulting in associated symptoms like headache, palpitations or flush. Non-functioning CBTs usually present as slowly and often tender growing mass which may affect adjacent cranial nerves. CBTs can be visualized by ultrasound, CT, MRI or angiography. Pre-surgical interventional embolization, surgical resection and radiation therapy are therapeutical options. The aim of this retrospective single-center study is to assess the safe and real-time evaluation of CBTs by contrast-enhanced ultrasound (CEUS).

Methods: Ten patients with CBT were included in this study on whom CEUS was performed between 2007-2018 (mean age: 62 years). In 6/10 patients, results were confirmed by MRI, 4/12 patients underwent subsequent angiography. CEUS was performed and interpreted by a single consultant radiologist with experience since 2000 (EFSUMB level 3). VueBox ${ }^{\circledR}$ software was used for standardized perfusion quantification.

Results: CEUS allowed to detect all CBTs and visualize intratumoral microcirculation. Perfusion quantification was performed in 6/10 cases. CBTs showed significantly reduced peak-enhancement (PE), reduced wash-in perfusion index (WiPI) and significantly elevated time to peak (TTP) compared to common carotid arteries (CCA).

Conclusions: CEUS is a useful and safe tool for identifying CBTs and evaluating intratumoral microperfusion at high spatial and temporal resolutions in real-time. In addition to conventional ultrasound, CT, MRI and digital substraction angiography (DSA), CEUS may be implemented in the future diagnostic work-up and follow-up of CBT patients.

Keywords: Carotid body tumor (CBT); diagnostic imaging; neck; neoplasms

Submitted Nov 03, 2019. Accepted for publication May 25, 2020.

doi: 10.21037/qims-19-920

View this article at: http://dx.doi.org/10.21037/qims-19-920 


\section{Introduction}

Carotid body tumor (CBT) depicts an extra-adrenal paraganglioma which arises from paraganglia that in turn form a neuroendocrine branch of the autonomous nervous system (1) ranging from skull base to the pelvic region. CBTs comprise more than $50 \%$ of the head and neck paraganglioma (2) and were reported to fulfill chemoreceptor function for the respiratory and cardiovascular system. Other localizations of paraganglioma are adjacent to the aortic arch, jugulotympanic, in the posterior mediastinum, abdominal paraaortic (including Zuckerkandl's body) or the adrenal medulla, the latter is known as pheochromocytoma. There is a preponderance for CBTs in women (women: men $=2: 1$ ) and they occur mainly in the 5th-6th decade (1). In $10 \%$ CBTs are found bilaterally. Most CBTs are benign tumors; in up to $15 \%$ of cases, metastases to liver, bone, lymph nodules or lungs are described $(3,4)$. Catecholamine secreting paraganglioma are so-called "functioning paraganglioma" and may induce symptoms like palpitations or headache (5). Non-functioning paranganglioma usually appears as a growing palpable mass often with tenderness. Due to the vicinity to cranial nerves $\mathrm{X}$-XII, patients may further develop hoarseness of voice, dysphagia or odynophagia (6). CBTs can either develop sporadically or hereditarily. It is estimated that up to $40 \%$ of head and neck paragangliomas are hereditary (7). Interestingly, the correlation between chronic hypoxia and incidence of CBTs was linked to pseudohypoxiaassociated genes of the succinate dehydrogenase enzyme (SDH) $(8,9)$. Among hereditary syndromes linked with CBTs are paraganglioma syndromes $1-5$, neurofibromatosis type I, tuberous sclerosis, Von-Hippel-Lindau syndrome, multiple endocrine neoplasia type $2(7,10)$.

CBTs can be visualized by conventional ultrasound, computed tomography (CT), magnetic resonance imaging (MRI) or by digital substraction angiography (DSA). In initial native B-mode, CBTs usually appear as hypoechoic formations. Hypervascularization can normally be demonstrated using color Doppler mode (11).

The Endocrine Society recommends CT and MR imaging as the imaging modalities of choice in the diagnostic work-up of CBTs (12). Upon application of i.v. contrast in CT and MRI, CBTs usually present homogeneous contrast enhancement due to their hypervascularization, but my also contain areas of necrosis and hemorrhage. CBTs are well-demarcated with densities equivalent to muscle tissue in CT scans. Typical morphological appearance on MRI is a low to iso T1-signal and a high T2-signal with a characteristic "salt-and-pepper" pattern (13). Angiographically, hypervascularization, feeding and draining vessels and tumor blush can be visualized $(1,14)$. Splaying of the internal and external carotid arteries and causing a lyre-like appearance is a feature indicating CBT. Besides the invasive nature, ectopic embolism, vascular ruptures and a high prevalence of cardiac arrest during angiographic assessment of CBTs were previously reported $(15,16)$. Before MRI and CT can be performed, thorough protocols need to be evaluated respecting potential comorbidities of the patients like renal impairment, thyroid gland disbalances, allergic reactions to contrast media in order to safeguard appropriate scans. Ionizing radiation in CT and angiography should also be considered. Moreover, patients may depend on medical devices like cardiac pacemaker which might be a contraindication for MRI. With an excellent safety profile (17), its distinct costeffectiveness and its direct accessibility, contrast-enhanced ultrasound (CEUS) depicts an alternative powerful imaging tool to visualize CBTs. Compared to CT and MRI, CEUS allows for real-time imaging at higher spatial and temporal resolutions. Another benefit of CEUS is that it can easily be repeated. The capability of CEUS to visualize intratumoral microperfusion had already been described in other solid tumors, like in gastrointestinal stromal tumors or in hepatocellular carcinoma (18-20). The fact that CBTs are highly vascularized tumors and the reported ability of CEUS for visualizing intratumoral microperfusion makes it an attractive and feasible diagnostic tool to analyze microcirculation within CBTs. It was already described, that CEUS is an appropriate instrument to detect intratumoral microperfusion and the extent of devascularization upon embolization in a single patient with a CBT, thus allowing for monitoring therapy efficiency (21).

Biopsies are very rarely requested in CBT patients due to high risk of bleeding and injury of the carotid artery (22).

Clinical management strategies for CBTs comprise initial wait-and-scan policy (23) and therapeutical options: presurgical interventional embolization, surgical resection and radiation therapy (24). The Shamblin classification for CBT which was introduced in 1971 assesses surgical resectability and differentiates three groups of CBT: group I tumors may be easily resected from the carotid arteries; group II tumors are more adjacent to the adventitia and partially surround the carotid bifurcation; group III tumors completely embrace the carotid bifurcation and are more adherent to the vessels (25).

In our present retrospective single-center study, 


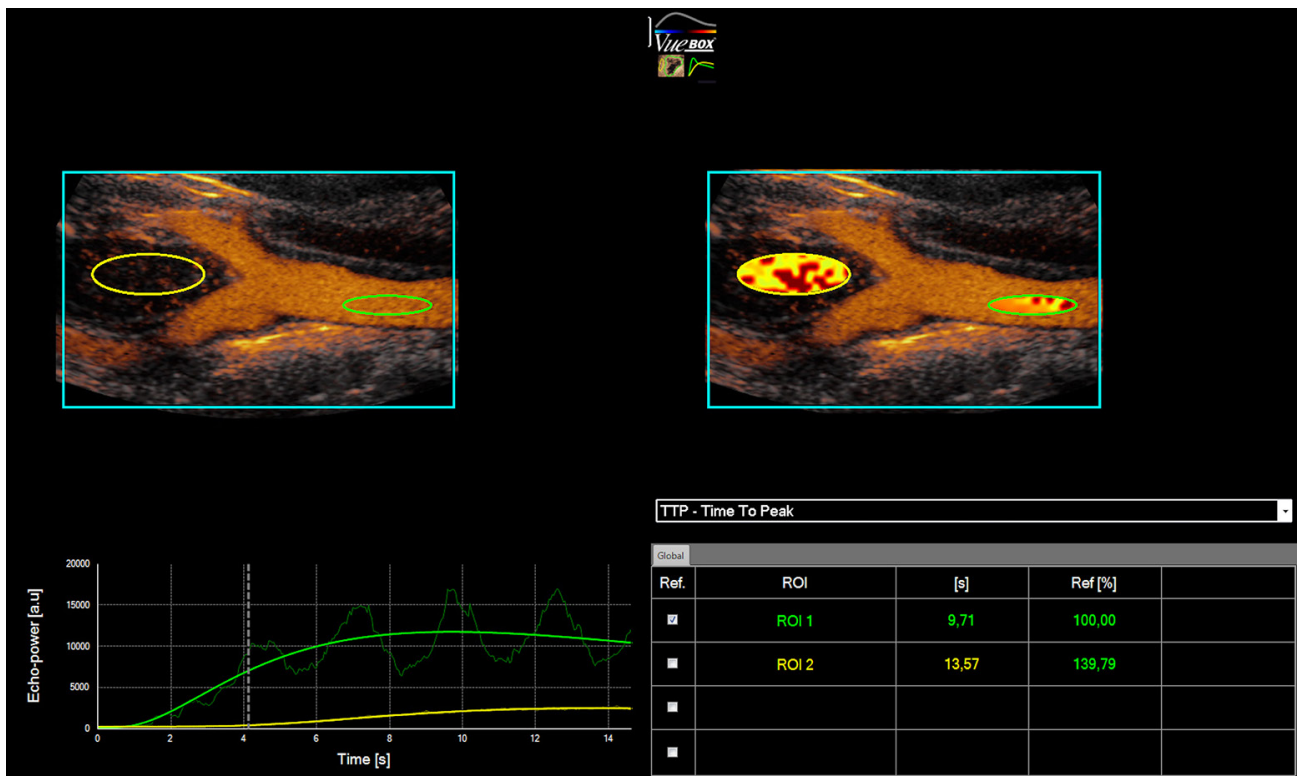

Figure 1 VueBox ${ }^{\circledR}$ user interface. Delimitation ROI illustrated as turquoise frames. ROI1 (green) in common carotid artery (CCA) as reference, ROI2 (yellow) in the carotid body tumor (CBT).

we evaluated the sonomorphological appearance and microperfusion of CBTs by CEUS.

\section{Methods}

This retrospective single-center study was approved by the local institutional ethical committee of the institutional review board and all contributing authors followed the ethical guidelines for publication in Quantitative Imaging in Medicine and Surgery (QIMS). All study data were gathered according to the principles expressed in the Declaration of Helsinki/(as revised in 2013). Oral and written informed consent of all patients was obtained before each CEUS examination and their associated risks and potential complications have been carefully described. All CEUS examinations were performed and analyzed by a single skilled radiologist with experience since 2000 (EFSUMB level 3). All included patients underwent native B-mode, color Doppler and CEUS scans in supine position. Up-to-date high-end ultrasound systems with adequate CEUS protocols were utilized (GE Healthcare LOGIQ L9, Chicago, Illinois, USA; Siemens Ultrasound Sequoia, ACUSON Sequoia, Mountain View, California, USA; Philips Ultrasound iU22, EPIQ 7, Seattle, USA). Appropriate linear transducers of the corresponding ultrasound systems were used: GE L92, Siemens L94, Philips L125. In every CEUS examination, a low mechanical index was used to avoid early destruction of microbubbles $(<0.2)$. For all CEUS examinations the second-generation blood pool contrast agent SonoVue ${ }^{\circledR}$ (Bracco, Milan, Italy) was used (17). 1.5-2.4 mL of SonoVue ${ }^{\circledR}$ were applied. After contrast agent was applied, a bolus of $5-10 \mathrm{~mL}$ sterile $0.9 \%$ sodium chloride solution was given. No adverse side effects upon administration of SonoVue ${ }^{\circledR}$ were registered. All CEUS examinations were successfully performed and image quality was sufficient in every single case. The patient files and imaging records were collected from the archiving system of our institution.

Perfusion quantification was performed using the quantification software VueBox ${ }^{\circledR}$ (Bracco Suisse SA-Software Applications, Geneva, Suisse) by using uncompressed and raw DICOM cine loops. After initial software calibration (depending on ultrasound transducers, presets, gain) regions of interest (ROI) were manually placed. Two observers set ROIs in consensus reading: firstly, the delimitation ROI, ROI1 into the common carotid artery (CCA) as reference and ROI2 into the CBT were set (Figure 1). ROIs did not change during the entire clip. Quantitative perfusion analysis was performed and parameters of special interest were peak-enhancement (PE), rise time (RT), time to peak (TTP), wash-in area under the curve (WiAUC), wash-in rate (WiR), wash-in perfusion index (WiPI). Due to the limited duration of the cine-loops and the retrospective character of this study, parameters for characterizing the 
Table 1 Clinical data of the included patients with carotid body tumor(s)

\begin{tabular}{|c|c|c|c|c|c|c|c|}
\hline Pat. No. & Age & Sex & Location & Max. diameter & CEUS & MRI & DSA \\
\hline \#2 & 74 & Female & Left & $2.6 \mathrm{~cm}$ & + & + & Embo. \\
\hline$\# 3$ & 42 & Female & Right & $3.0 \mathrm{~cm}$ & + & - & - \\
\hline \#4 & 32 & Male & Bilateral & Right: $5.3 \mathrm{~cm}$, left: $1.2 \mathrm{~cm}$ & + (CEUS only from left) & + & Embo. (right) \\
\hline \#6 & 57 & Female & Left & $4.0 \mathrm{~cm}$ & + & + & Embo. \\
\hline$\# 7$ & 46 & Male & Right & $1.2 \mathrm{~cm}$ & + & + & - \\
\hline$\# 8$ & 71 & Female & Right & $2.7 \mathrm{~cm}$ & + & + & - \\
\hline \#9 & 70 & Female & Bilateral & Right: $3.6 \mathrm{~cm}$, left: $3.1 \mathrm{~cm}$ & + & + & - \\
\hline
\end{tabular}

DSA, digital substraction angiography; Embo., embolization; CEUS, contrast-enhanced ultrasound; MRI, magnetic resonance imaging; No., number.

wash-out phase could not sufficiently be analyzed.

Statistical analysis was performed by using Graph Pad Prism (GraphPad Software Inc., La Jolla, USA). Wilcoxon's test was used for comparing quantified perfusion data. Statistical tests were considered significant if $\mathrm{P}$ value was $<0.05$.

A total of 10 patients on whom CEUS was performed between 2007-2018 were included in this retrospective single-center study.

\section{Results}

CEUS was successfully performed in all included patients without occurrence of any adverse effects. SonoVue ${ }^{\circledR}$ was used in each examination. Since it is a purely intravascular contrast agent that does not diffuse into the interstitial space, it thus allows for dynamic assessment of microcirculation. The mean age of the included 10 patients at the time of CEUS performance was 62 years (range: 32-88 years) of whom 3 were men and 7 women (ratio men:women =1:2.3) (Table 1). The mean size of the CBTs was $2.7 \mathrm{~cm}$ (range: $1.2-4.0 \mathrm{~cm}$ ), of which 5 were located left and 3 on the right side. In 2 cases bilateral CBTs occurred. Six patients underwent additional MRI scans. Four patients underwent successful interventional embolization of the CBT $\left(50 \%\right.$ injection of Onyx ${ }^{\circledR}, 50 \%$ application of microcoils).

CEUS could detect CBTs and visualize intratumoral microperfusion at high spatial and temporal resolutions in real-time in all cases (a representative case is illustrated in Figure $2 A, B, C)$. Due to disturbing moving artifacts, perfusion quantification using VueBox ${ }^{\circledR}$ could only be sufficiently performed in 6 of the 10 patients. Comparing quantitative perfusion data of CBTs with adjacent CCAs showed that CBTs featured significantly reduced PE, significantly reduced WiPI and significantly elevated TTP (Figure 3). The analysis of RT, WiR and WiAUC did not elaborate significant differences between CCAs and CBTs: Tendencies for elevated RT, reduced WiR and reduced WiAUC in CBTs in comparison with CCAs were registered.

\section{Discussion}

Ultrasound is the imaging modality of choice when it comes to pathologies of the carotid arteries (26). Noteworthy, ultrasound showed high diagnostic accuracy for evaluating the severity of stenosis and occlusion of internal carotid artery (ICA) (27). Furthermore, CEUS was described as a helpful instrument for evaluating in-stent restenosis after carotid stenting of the ICA (28) and carotid dissection (29). By means of CEUS, microbubbles at the base of carotid plaques could be detected; the corresponding histopathological analysis revealed enhanced vascular endothelial growth factor (VEGF) expression as a surrogate parameter for neoangiogenesis by which differentiation between symptomatic and asymptomatic plaques might be feasible (30). 

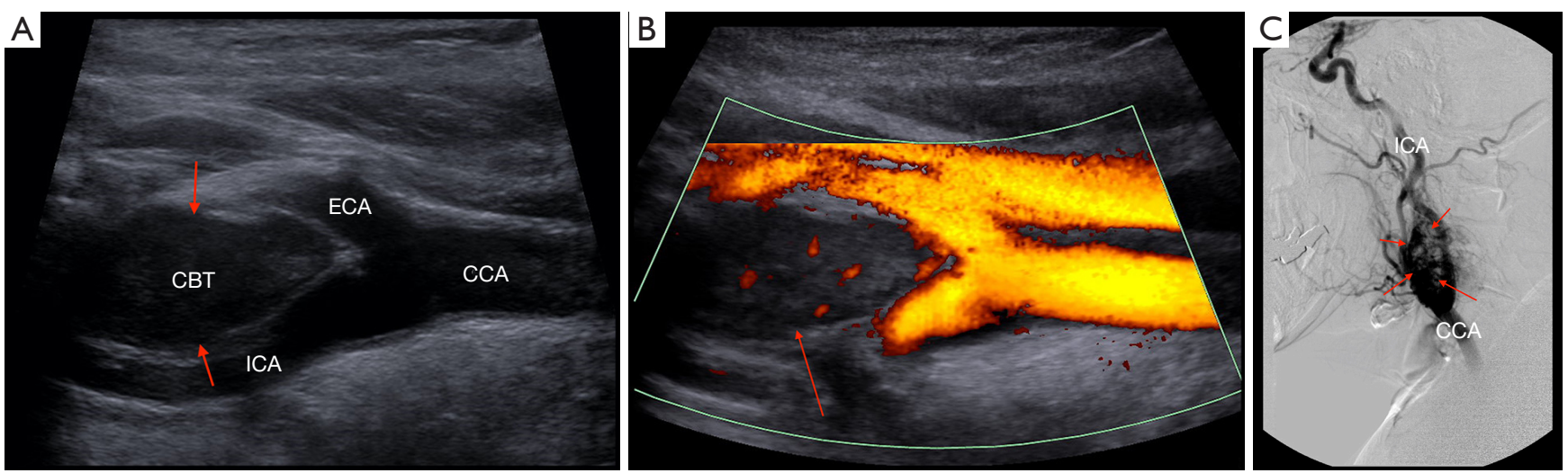

Figure 2 Sonomorphological appearance of carotid body tumor (CBT). (A) Mainly hypoechoic and slightly inhomogeneous mass within the left carotid bifurcation. The carotid body tumor is delineated by red arrows. (B) Slight hypervascularization within the CBT detected via power Doppler mode. The carotid body tumor is delineated by the red arrow. (C) Correlative appearance of the carotid body tumor (CBT) in digital substraction angiography. The CBT is delineated by red arrows. CCA, common carotid artery; ICA, internal carotid artery; ECA, external carotid artery; CBT, carotid body tumor.
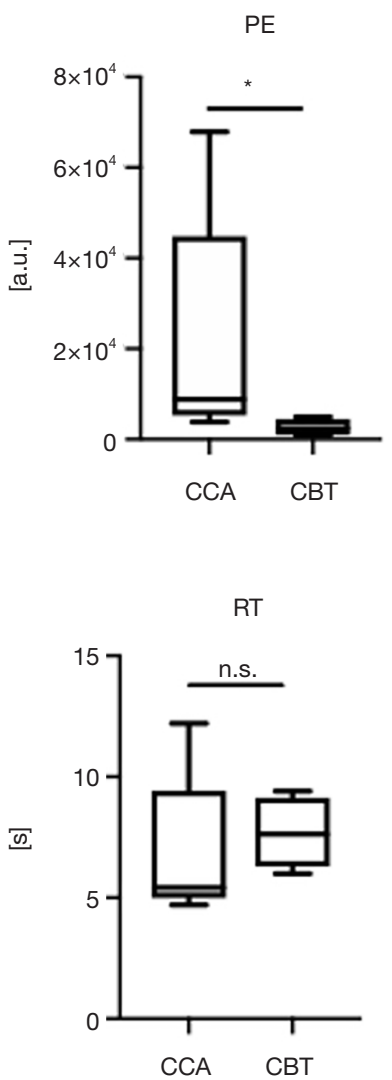
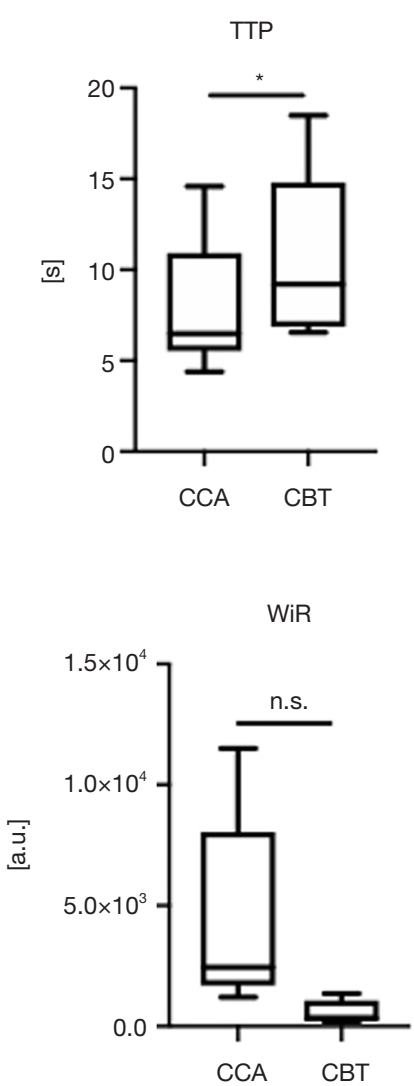
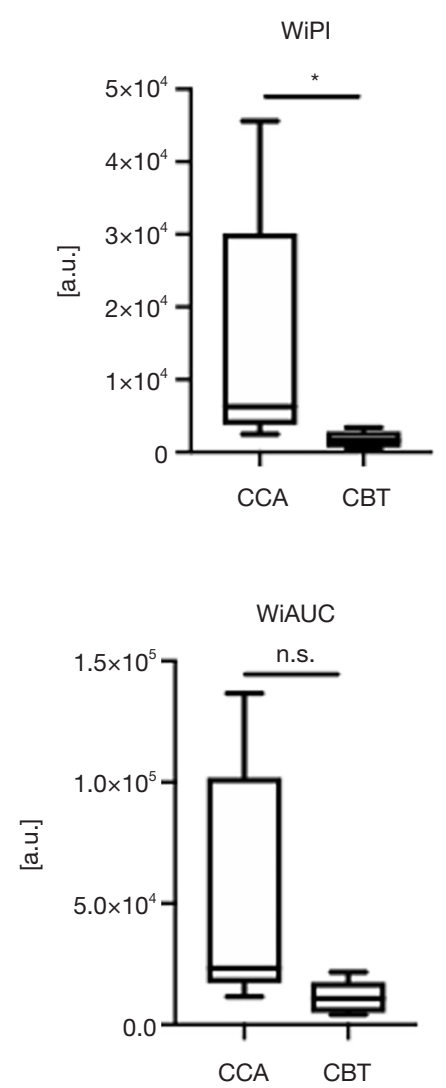

Figure 3 Boxplots comparing quantitative perfusion parameters between CBT and CCA, n=6. * $\mathrm{P}<0.05$. PE, peak-enhancement; TTP, time to peak; WiPI, wash-in perfusion index; RT, rise time; WiR, wash-in rate; WiAUC, wash-in area under the curve; a.u., arbitrary units; s, seconds; n.s., not significant. CCA, common carotid artery; CBT, carotid body tumor. 
$\mathrm{CT}$ and MR angiography depict the non-invasive imaging modalities of choice to detect CBTs $(12,31)$. Due to associated ionizing radiation, CT angiography should be thoroughly evaluated. The value of CT and MRI might be affected in case of disturbing metal artifacts e.g. dental implants (32). Pre-operative assessment of the vasculature, intratumoral calcifications and bone destructions as in the case of skull base tumors are of value in the frame of CT scans. Conventional substraction angiography is useful for pre-operative diagnostic and for therapeutical embolization. Metabolic imaging like somatostatin receptor scintigraphy (SRS) and $\left[{ }^{18} \mathrm{~F}\right]$-FDOPA positron emission tomography (PET) allow for whole body scans which might be critical for staging of multifocal tumor disease $(33,34)$.

Sonography is the first-line instrument allowing for immediately accessible delineation of CBTs and shows a high diagnostic accuracy in identifying CBTs (11). CBTs appear inhomogeneously hypoechoic in native B-mode and show hypervascularization in Color Doppler due to their vascularized nature. Quantitative perfusion analysis using CEUS has already been described for different disease entities like prostate cancer (35), hepatocellular and cholangiocellular cancer (36), autoimmune pancreatitis (37) or Crohn's disease (38). Previously, CEUS was already reported to be an eligible tool to monitor intratumoral microperfusion and its devascularization upon percutaneous embolization of a CBT $(21,26)$ in the frame of a case report. Besides, there has not been any reported study to further illustrate the role of CEUS for assessing CBTs.

To our knowledge, this present retrospective singlecenter study is the first using CEUS to scrutinize and quantify intratumoral microperfusion of CBTs. We could show that CEUS is a reliable diagnostic tool to detect CBTs, and other than CT, MRI or angiography allows for visualization of intratumoral microperfusion at high spatial and temporal resolutions. CEUS allows for excluding other potential differentials, e.g. hemangioma, metastasis or branchial cleft cysts. Of value here is the fact that CEUS depicts a safe, non-ionizing, cost-effective and easily accessible instrument that is also repeatable with less precautions to take than for CT and MRI. The abovementioned artifacts due to in-situ metal devices in CT and MRI do not occur in CEUS. Moreover, CEUS can safely be applied even in children and during pregnancy $(39,40)$.

The vascular nature of CBTs is reflected in the presented perfusion quantification parameters: All included CBTs showed contrast enhancement. Nonetheless, since CBTs are characteristically comprised of dense neoplastic cell nests ("zellballen") with surrounding stromal component and are not sole vessels (10), significantly reduced PE, significantly reduced WiPI and significantly elevated TTP were registered (Figure 3). Indicated tendencies for prolonged RT, reduced WiR and reduced WiAUC in CBTs juxtaposed to corresponding CCAs could be observed which might be explained by the heterogeneity and the limited number of included patients. The present study should be regarded as a proof of concept, namely that CEUS facilitates for dynamic assessment of microperfusion in CBTs. Still, for an entire characterization of the microperfusion dynamics of CBTs, wash-out perfusion parameters need to be investigated within the frame of future studies.

Whereas intravenous CT and MRI contrast agents extravasate from the (tumor) vessels into the interstitial environment, as previously shown in the published case report in 2015 (21), CEUS is capable of visualizing and evaluating microperfusion of CBTs in the first place and monitoring its alteration upon embolization to evaluate therapeutical outcome ("functional imaging"). Up to date, there is no official recommendation neither by the World Federation for Ultrasound in Medicine and Biology (WFUMB), nor by the European Federation of Societies for Ultrasound in Medicine and Biology (EFSUMB) nor by endocrine/ear nose and throat (ENT) societies (41) for deploying CEUS in the diagnostic work-up of CBTs.

\section{Conclusions}

The high diagnostic sensitivity of CEUS allows for measuring even faint intratumoral microperfusion-and beyond-in a real-time manner, unlike other imaging modalities like CT and MRI, at high spatial and temporal resolutions, the possibility to save cine-loops with an excellent safety profile; thus CEUS might be of critical value in the future diagnostic work-up and follow-up of CBT patients in the clinical routine. Further clinical (prospective) studies need to validate the presented results, elaborate cut off values for perfusion parameters and establish a clinical algorithm in which integrated CEUS is a solid component.

Limitations of the present retrospective single-center study is its limited number of included patients and differing ultrasound systems.

\section{Acknowledgments}

Funding: None. 


\section{Footnote}

Conflicts of Interest: All authors have completed the ICMJE uniform disclosure form (available at http://dx.doi. org/10.21037/qims-19-920). The authors have no conflicts of interest to declare.

Ethical Statement: The study was conducted in accordance with the Declaration of Helsinki (as revised in 2013). This retrospective single-center study was approved by the local institutional ethical committee of the institutional review board and all contributing authors followed the ethical guidelines for publication in Quantitative Imaging in Medicine and Surgery (QIMS). Oral and written informed consent of all patients was obtained before each CEUS examination and their associated risks and potential complications have been carefully described.

Open Access Statement: This is an Open Access article distributed in accordance with the Creative Commons Attribution-NonCommercial-NoDerivs 4.0 International License (CC BY-NC-ND 4.0), which permits the noncommercial replication and distribution of the article with the strict proviso that no changes or edits are made and the original work is properly cited (including links to both the formal publication through the relevant DOI and the license). See: https://creativecommons.org/licenses/by-nc-nd/4.0/.

\section{References}

1. Lee KY, Oh YW, Noh HJ, Lee YJ, Yong HS, Kang EY, Kim KA, Lee NJ. Extraadrenal paragangliomas of the body: imaging features. AJR Am J Roentgenol 2006; 187:492-504.

2. Rao AB, Koeller KK, Adair CF. From the archives of the AFIP. Paragangliomas of the head and neck: radiologicpathologic correlation. Armed Forces Institute of Pathology. Radiographics 1999;19:1605-32.

3. Jimenez C, Rohren E, Habra MA, Rich T, Jimenez P, Ayala-Ramirez M, Baudin E. Current and future treatments for malignant pheochromocytoma and sympathetic paraganglioma. Curr Oncol Rep 2013;15:356-71.

4. Corssmit EP, Romijn JA. Clinical management of paragangliomas. Eur J Endocrinol 2014;171:R231-43.

5. Yeo H, Roman S. Pheochromocytoma and functional paraganglioma. Curr Opin Oncol 2005;17:13-8.

6. Davidovic LB, Djukic VB, Vasic DM, Sindjelic RP,
Duvnjak SN. Diagnosis and treatment of carotid body paraganglioma: 21 years of experience at a clinical center of Serbia. World J Surg Oncol 2005;3:10.

7. Williams MD. Paragangliomas of the Head and Neck: An Overview from Diagnosis to Genetics. Head Neck Pathol 2017;11:278-87.

8. Rodríguez-Cuevas S, Lopez-Garza J, LabastidaAlmendaro S. Carotid body tumors in inhabitants of altitudes higher than 2000 meters above sea level. Head Neck 1998;20:374-8.

9. Boedeker CC, Hensen EF, Neumann HP, Maier W, van Nederveen FH, Suarez C, Kunst HP, Rodrigo JP, Takes RP, Pellitteri PK, Rinaldo A, Ferlito A. Genetics of hereditary head and neck paragangliomas. Head Neck 2014;36:907-16.

10. Wieneke JA, Smith A. Paraganglioma: carotid body tumor. Head Neck Pathol 2009;3:303-6.

11. Demattè S, Di Sarra D, Schiavi F, Casadei A, Opocher G. Role of ultrasound and color Doppler imaging in the detection of carotid paragangliomas. J Ultrasound 2012;15:158-63.

12. Lenders JW, Duh QY, Eisenhofer G, GimenezRoqueplo AP, Grebe SK, Murad MH, Naruse M, Pacak K, Young WF Jr, Endocrine S. Pheochromocytoma and paraganglioma: an endocrine society clinical practice guideline. J Clin Endocrinol Metab 2014;99:1915-42.

13. Olsen WL, Dillon WP, Kelly WM, Norman D, Brant-Zawadzki M, Newton TH. MR imaging of paragangliomas. AJR Am J Roentgenol 1987;148:201-4.

14. Hesselink JR, Davis KR, Taveras JM. Selective arteriography of glomus tympanicum and jugulare tumors: techniques, normal and pathologic arterial anatomy. AJNR Am J Neuroradiol 1981;2:289-97.

15. Hua Q, Xu Z, Jiang Y. Diagnosis and surgical treatment of carotid body tumor: A retrospective analysis of 58 patients. Oncol Lett 2017;14:3628-32.

16. Power AH, Bower TC, Kasperbauer J, Link MJ, Oderich G, Cloft H, Young WF Jr, Gloviczki P. Impact of preoperative embolization on outcomes of carotid body tumor resections. J Vasc Surg 2012;56:979-89.

17. Piscaglia F, Bolondi L, Italian Society for Ultrasound in M, Biology Study Group on Ultrasound Contrast A. The safety of Sonovue in abdominal applications: retrospective analysis of 23188 investigations. Ultrasound Med Biol 2006;32:1369-75.

18. Lassau N, Chami L, Benatsou B, Peronneau P, Roche A. Dynamic contrast-enhanced ultrasonography (DCE-US) with quantification of tumor perfusion: a new diagnostic 
tool to evaluate the early effects of antiangiogenic treatment. Eur Radiol 2007;17 Suppl 6:F89-98.

19. Lassau N, Lamuraglia M, Chami L, Leclere J, Bonvalot S, Terrier P, Roche A, Le Cesne A. Gastrointestinal stromal tumors treated with imatinib: monitoring response with contrast-enhanced sonography. AJR Am J Roentgenol 2006; 187:1267-73.

20. Yang D, Li R, Zhang XH, Tang CL, Ma KS, Guo DY, Yan XC. Perfusion Characteristics of Hepatocellular Carcinoma at Contrast-enhanced Ultrasound: Influence of the Cellular differentiation, the Tumor Size and the Underlying Hepatic Condition. Sci Rep 2018;8:4713.

21. Rübenthaler J, Lutz J, Reiser M, Clevert DA. Title Page - Paraganglioma of the Head and Neck: Follow-Up of Interventional Procedures with CEUS. Ultraschall Med 2015;36:541-3.

22. Rosa M, Sahoo S. Bilateral carotid body tumor: the role of fine-needle aspiration biopsy in the preoperative diagnosis. Diagn Cytopathol 2008;36:178-80.

23. Jansen TTG, Timmers H, Marres HAM, Kunst HPM. Feasibility of a wait-and-scan period as initial management strategy for head and neck paraganglioma. Head Neck 2017;39:2088-94.

24. Dupin C, Lang P, Dessard-Diana B, Simon JM, Cuenca X, Mazeron JJ, Feuvret L. Treatment of head and neck paragangliomas with external beam radiation therapy. Int J Radiat Oncol Biol Phys 2014;89:353-9.

25. Shamblin WR, ReMine WH, Sheps SG, Harrison EG, Jr. Carotid body tumor (chemodectoma). Clinicopathologic analysis of ninety cases. Am J Surg 1971;122:732-9.

26. Clevert DA, Paprottka P, Sommer WH, Helck A, Reiser MF, Zengel P. The role of contrast-enhanced ultrasound in imaging carotid arterial diseases. Semin Ultrasound CT MR 2013;34:204-12.

27. Grant EG, Benson CB, Moneta GL, Alexandrov AV, Baker JD, Bluth EI, Carroll BA, Eliasziw M, Gocke J, Hertzberg BS, Katanick S, Needleman L, Pellerito J, Polak JF, Rholl KS, Wooster DL, Zierler RE. Carotid artery stenosis: gray-scale and Doppler US diagnosis--Society of Radiologists in Ultrasound Consensus Conference. Radiology 2003;229:340-6.

28. Clevert DA, Sommer WH, Helck A, Reiser M. Duplex and contrast enhanced ultrasound (CEUS) in evaluation of in-stent restenosis after carotid stenting. Clin Hemorheol Microcirc 2011;48:199-208.

29. Clevert DA, Sommer WH, Zengel P, Helck A, Reiser M. Imaging of carotid arterial diseases with contrast-enhanced ultrasound (CEUS). Eur J Radiol 2011;80:68-76.
30. Giannoni MF, Vicenzini E, Citone M, Ricciardi MC, Irace L, Laurito A, Scucchi LF, Di Piero V, Gossetti B, Mauriello A, Spagnoli LG, Lenzi GL, Valentini FB. Contrast carotid ultrasound for the detection of unstable plaques with neoangiogenesis: a pilot study. Eur J Vasc Endovasc Surg 2009;37:722-7.

31. van den Berg R, Verbist BM, Mertens BJ, van der Mey AG, van Buchem MA. Head and neck paragangliomas: improved tumor detection using contrast-enhanced 3D time-of-flight MR angiography as compared with fat-suppressed MR imaging techniques. AJNR Am J Neuroradiol 2004;25:863-70.

32. Klinke T, Daboul A, Maron J, Gredes T, Puls R, Jaghsi A, Biffar R. Artifacts in magnetic resonance imaging and computed tomography caused by dental materials. PLoS One 2012;7:e31766.

33. Treglia G, Cocciolillo F, de Waure C, Di Nardo F, Gualano MR, Castaldi P, Rufini V, Giordano A. Diagnostic performance of $18 \mathrm{~F}$-dihydroxyphenylalanine positron emission tomography in patients with paraganglioma: a meta-analysis. Eur J Nucl Med Mol Imaging 2012;39:1144-53.

34. Guichard JP, Fakhry N, Franc J, Herman P, Righini CA, Taieb D. Morphological and functional imaging of neck paragangliomas. Eur Ann Otorhinolaryngol Head Neck Dis 2017;134:243-8.

35. Maxeiner A, Fischer T, Schwabe J, Baur ADJ, Stephan C, Peters R, Slowinski T, von Laffert M, Marticorena Garcia SR, Hamm B, Jung EM. Contrast-Enhanced Ultrasound (CEUS) and Quantitative Perfusion Analysis in Patients with Suspicion for Prostate Cancer. Ultraschall Med 2019;40:340-8.

36. Wildner D, Pfeifer L, Goertz RS, Bernatik T, Sturm J, Neurath MF, Strobel D. Dynamic contrast-enhanced ultrasound (DCE-US) for the characterization of hepatocellular carcinoma and cholangiocellular carcinoma. Ultraschall Med 2014;35:522-7.

37. Vitali F, Pfeifer L, Janson C, Goertz RS, Neurath MF, Strobel D, Wildner D. Quantitative perfusion analysis in pancreatic contrast enhanced ultrasound (DCE-US): a promising tool for the differentiation between autoimmune pancreatitis and pancreatic cancer. Z Gastroenterol 2015;53:1175-81.

38. Girlich C, Jung EM, Iesalnieks I, Schreyer AG, Zorger N, Strauch U, Schacherer D. Quantitative assessment of bowel wall vascularisation in Crohn's disease with contrast-enhanced ultrasound and perfusion analysis. Clin Hemorheol Microcirc 2009;43:141-8. 
39. Seitz K, Strobel D. A Milestone: Approval of CEUS for Diagnostic Liver Imaging in Adults and Children in the USA. Ultraschall Med 2016;37:229-32.

40. Schwarze V, Marschner C, Negrao de Figueiredo G, Rubenthaler J, Clevert DA. Single-Center Study: Evaluating the Diagnostic Performance and Safety of Contrast-Enhanced Ultrasound (CEUS) in Pregnant Women to Assess Hepatic Lesions. Ultraschall Med 2020;41:29-35.

41. Sidhu PS, Cantisani V, Dietrich CF, Gilja OH, Saftoiu
A, Bartels E, Bertolotto M, Calliada F, Clevert DA, Cosgrove D, Deganello A, D'Onofrio M, Drudi FM, Freeman S, Harvey C, Jenssen C, Jung EM, Klauser AS, Lassau N, Meloni MF, Leen E, Nicolau C, Nolsoe C, Piscaglia F, Prada F, Prosch H, Radzina M, Savelli L, Weskott HP, Wijkstra H. The EFSUMB Guidelines and Recommendations for the Clinical Practice of Contrast-Enhanced Ultrasound (CEUS) in Non-Hepatic Applications: Update 2017 (Short Version). Ultraschall Med 2018;39:154-80.

Cite this article as: Schwarze V, Marschner C, Negrao De Figueiredo G, Ingrisch M, Rübenthaler J, Clevert DA. Singlecenter study: dynamic contrast-enhanced ultrasound in the diagnostic assessment of carotid body tumors. Quant Imaging Med Surg 2020;10(9):1739-1747. doi: 10.21037/qims-19-920 